IDDF2021-ABS-0047 ASSOCIATION OF ALBI GRADE, APRI SCORE AND ALBI-APRI SCORE WITH POSTOPERATIVE OUTCOMES AMONG PATIENTS WITH LIVER CIRRHOSIS AFTER NONHEPATIC SURGERY

Lorenz Kristoffer Daga*. National Kidney and Transplant Institute, Philippines

\subsection{6/gutjnl-2021-IDDF.71}

Background Patients with liver cirrhosis have an increased risk for poor postoperative outcomes after nonhepatic surgery, with liver dysfunction being the most important predictor of poor outcomes. This study aims to determine the association of the albumin-bilirubin (ALBI) grade, aspartate aminotransferase-platelet ratio index (APRI) score and ALBI-APRI score with postoperative outcomes among cirrhotic patients who have undergone nonhepatic surgery.

Methods This was a retrospective cohort study involving 34 patients. Age, ASA class, urgency of surgery, etiology of liver cirrhosis, preoperative Child-Turcotte-Pugh (CTP) score, Model for End-Stage Liver Disease (MELD) score, ALBI grade, APRI score and ALBI-APRI score were documented. The outcomes analyzed were postoperative hepatic decompensation (POHD) and in-hospital mortality. Bivariate analysis using the MannWhitney U test and Fisher's exact test was performed. Receiver operating characteristic (ROC) curve analysis was performed to compare the ability of the liver scoring systems to predict the occurrence of study outcomes. Binary logistic regression was performed to measure the odds ratio.

Results The ALBI grade and ALBI-APRI score were significantly associated with both POHD and in-hospital mortality. Both scores were non-inferior to the CTP and MELD scores in predicting study outcomes. Compared to CTP and MELD scores, the ALBI grade is more sensitive but less specific in predicting POHD and as sensitive but more specific in predicting in-hospital mortality. The ALBI-APRI score was less sensitive but more specific than the ALBI grade in predicting both POHD and in-hospital mortality.

Conclusions The ALBI grade and ALBI-APRI score were both associated with postoperative hepatic decompensation and inhospital mortality and were non-inferior to the CTP score and MELD score in predicting short-term in-hospital outcomes among cirrhotic patients after nonhepatic surgery.

\section{IDDF2021-ABS-0050 VALIDATION OF PLATELET-ALBUMIN- BILIRUBIN (PALBI) SCORE FOR PREDICTING OVERALL SURVIVAL OF HEPATOCELLULAR CARCINOMA}

Minh Duc Pham, Ngoc Anh Tran*. Hanoi Medical University, Vietnam

\subsection{6/gutjnl-2021-IDDF.72}

Background To validate PALBI score for predicting overall survival of hepatocellular carcinoma.

Methods In a retrospective study from June 2017, 67 HCC patients at Hanoi Medical University Hospital were collected and followed up median overall survival until June 2021. The Platelets-Albumin-Bilirubin (PALBI) score was calculated based on three indexes: platelets, albumin, and total bilirubin. PALBI score was classified into three groups: PALBI-1, PALBI-2, and PALBI-3. PALBI score, Child-Pugh classification, and BCLC classification were also calculated to compare the predictive value by the receiver operating curves (ROC).
Results This study included 67 HCC patients, of which the proportion of males is 92.5 . The median age of the research group is 59 years. Hepatitis B accounts for $80.6 \%$ of HCC patients. The median overall survival of PALBI-1, PALBI-II, PALBI-III is 35.14 months, 28.25 months, and 10.14 months, respectively. The difference in overall survival among the three groups was statistically significant meaning, $p=0.000$. Additionally, ROC analysis in the entire cohort revealed that the PALBI score had better AUC than the Child-Pugh score and BCLC classification. In HCC patients who have Child-Pugh A classification, the PALBI score divided into 3 groups which have statistically significant differences.

Conclusions These results suggested that the PALBI score could be an alternative hepatic function score for stratifying HCC patients.

\section{IDDF2021-ABS-0053 CHINESE HERBAL MEDICINE FOR FUNCTIONAL DYSPEPSIA: NETWORK META- ANALYSIS OF PROKINETIC-CONTROLLED RANDOMISED TRIALS}

${ }^{1}$ Leonard Ho* ${ }^{2}$ Claire Chenwen Zhong, ${ }^{2}$ Charlene Hoi Lam Wong, ${ }^{3}$ Justin Che Yuen Wu ${ }^{4}$ Karina Kar-Han Chan, ${ }^{1}$ Irene Xinyin Wu. 'School of Chinese Medicine, Faculty of Medicine, The Chinese University of Hong Kong, Hong Kong; ${ }^{2}$ The Jockey Club School of Public Health and Primary Care, Faculty of Medicine, The Chinese University of Hong Kong, Hong Kong; ${ }^{3}$ Department of Medicine and Therapeutics, Faculty of Medicine, The Chinese University of Hong Kong, Hong Kong; ${ }^{4}$ United Christian Nethersole Community Health Service - The Chinese University of Hong Kong Chinese Medicine Clinic cum Training and Research Centre (Tai Po District), Hong Kong

\subsection{6/gutjnl-2021-IDDF.73}

Background Functional dyspepsia (FD) is a common functional gastrointestinal disorder, with a prevalence of $5-30 \%$ among Asian countries. Prokinetic is the first-line conventional treatment in Asia, despite potential adverse events ranging from dystonia to life-threatening arrhythmia. Chinese herbal medicine (CHM) was mentioned in the Asian Consensus Report on Functional Dyspepsia as a potential treatment option. This network meta-analysis (NMA) aimed to evaluate the comparative effectiveness of different CHM formulae for FD against prokinetics.

Methods Four Chinese (Wanfang Data, China National Knowledge Infrastructure, SinoMed, and Index to Taiwan Periodical Literature System) and three international (MEDLINE, EMBASE, and Cochrane Central Register of Controlled Trials) databases were searched for randomised controlled trials (RCTs) on CHM versus prokinetics. The risk of bias among RCTs was assessed using Cochrane Risk-of-Bias Tool 2. Data from RCTs were obtained for random-effect pairwise metaanalyses. Random-effect NMAs were carried out to evaluate the comparative effectiveness of CHM formulae. GRADE partially contextualised framework was applied for NMA result interpretation.

Results Twenty-six different CHM formulae were identified from twenty-eight RCTs of mediocre quality. Pairwise metaanalyses indicated that $\mathrm{CHM}$ was superior to prokinetics in alleviating global symptoms at 4-week follow-up (pooled risk difference (RD): $0.14 ; 95 \%$ confidence interval (CI): 0.10 0.19 ), even after trim and fill adjustment for publication bias. Nevertheless, the effect size failed to exceed the minimally clinically important difference of 0.20 RD. NMAs demonstrated that Modified Zhi Zhu Decoction may have a moderate beneficial effect on alleviating global symptoms at 4-week 
follow-up (RD: 0.28; 95\% CI: -0.03-0.75). Xiao Pi Kuan Wei Decoction may have a large beneficial effect on alleviating postprandial fullness (Standardised mean difference (SMD): 2.14; 95\% CI: $-2.76-0.70$ ), early satiety (SMD: $-3.90 ; 95 \%$ CI: $-0.68--0.42$ ), and epigastric pain (SMD: -1.23 ; 95\% CI: -1.66- -0.29). No serious adverse events were reported.

Conclusions Modified Zhi Zhu Decoction and Xiao Pi Kuan Wei Decoction may be considered as an alternative for patients unresponsive to prokinetics. Confirmatory head-tohead trials should be conducted to investigate their comparative effectiveness against prokinetics.

\section{IDDF2021-ABS-0056 LIVER STIFFNESS PLUS PLATELETS PREDICTS LONG-TERM OUTCOMES IN CHILD-PUGH A CIRRHOSIS}

${ }^{1}$ Tanya Lee* ${ }^{2}$ Elliot Freeman, ${ }^{2}$ Daniel Clayton-Chub, ${ }^{2}$ Stuart Roberts, ${ }^{1}$ Nik Ding, ${ }^{2}$ William Kemp, ${ }^{2}$ Rohit Sawhney, ${ }^{2} J o h n$ Lubel, ${ }^{1}$ David Iser, ${ }^{1}$ Jessica Howell, ${ }^{1}$ Catherine Croagh, ${ }^{1}$ Thai Hong, 'Barbara Demediuk, ${ }^{1}$ Paul Desmond, ' 5 wee Lin Chen Yi Mei, ${ }^{1}$ Alexander Thompson, ${ }^{1}$ Jacinta Holmes. 'St Vincent's Hospital, Australia; ${ }^{2}$ Alfred Health, Australia

\subsection{6/gutjnl-2021-IDDF.74}

Background Portal hypertension (PH) remains an important sequela of cirrhosis, contributing to morbidity and mortality. Non-invasive algorithms identifying patients at risk of clinically significant portal hypertension (CSPH) and high-risk gastrooesophageal varices (varices needing treatment, VNT) are critical. We previously described an algorithm whereby liver stiffness measurement (LSM) $\leq 25 \mathrm{kPa}$ plus platelets $\geq 100$ excluded VNT in Child-Pugh A cirrhosis. 5 years following the inception of our initial study, this study aimed to assess the prognostic value of $\mathrm{LSM} \leq 25 \mathrm{kPa}$ plus platelets $\geq 100$ in predicting long-term hepatic decompensation and death.

Methods This is a retrospective multicentre study of patients from two tertiary centres. Medical records were reviewed for all subsequent gastroscopies, LSM, six-monthly laboratory tests and abdominal ultrasounds, symptoms/signs consistent with hepatic decompensation (ascites, variceal bleeding, hepatic encephalopathy), and mortality from the initial study date. Patients with an LSM $>25 \mathrm{kPa}$ plus platelets $<100$ were considered high risk for VNT, while patients with $\mathrm{LSM} \leq 25 \mathrm{kPa}$ and/or platelets $\geq 100$ were considered low risk.

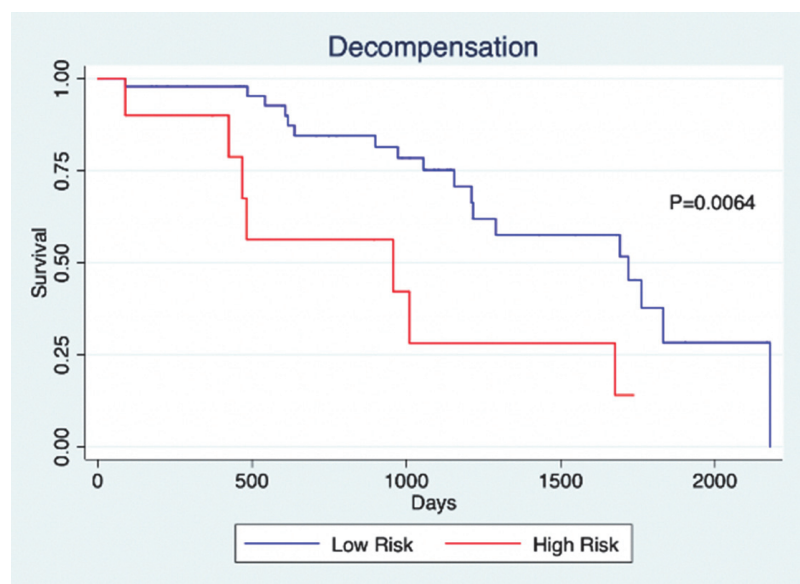

Abstract IDDF2021-ABS-0056 Figure 1

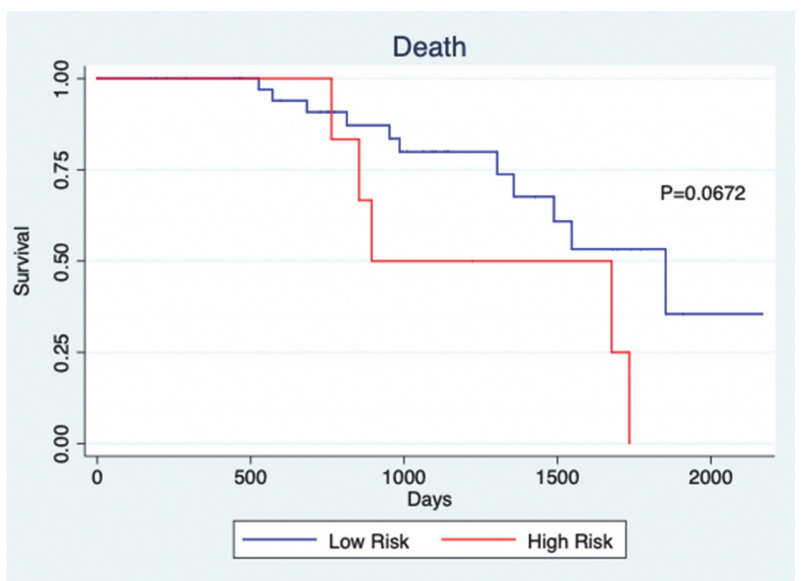

Abstract IDDF2021-ABS-0056 Figure 2

Results 180 patients were included. Median age was 55 yrs (IQR 49-63), 70\% male, with no significant difference between groups. Median follow-up was 7.7 yrs (4.5-9.0). At study inception, 30/180 (17\%) were high risk. The majority $(114 / 180,63 \%)$ had cirrhosis secondary to HCV, similar according to risk group $(\mathrm{p}=0.752)$. Decompensation was significantly more common in high-risk patients (11/30 (37\%) vs 26/150 (17\%) respectively, $p=0.016$; (IDDF2021-ABS-0056 Figure 1. Decompensation according to high-risk and low-risk category)). 2/150 (1\%) low-risk patients were re-classified as high-risk. 19/150 (13\%) of low-risk patients had VNT compared to $7 / 30(23 \%)$ in the high-risk group $(p=0.043)$. There was a trend towards higher mortality in high-risk vs low-risk patients $(12 / 30(40 \%)$ vs $36 / 150$ (24\%), respectively, $p=0.067$; (IDDF2021-ABS-0056 Figure 2. Death according to high-risk and low-risk category)).

Conclusions Our previously defined algorithm of LSM and platelets has prognostic value in predicting for subsequent decompensation and death. LSM+PLT can assist in stratifying future risk of liver-related events in Child-Pugh A cirrhotics.

\section{IDDF2021-ABS-0064 ASCITIC FLUID TOTAL CELL COUNT AND POLYMORPHONUCLEAR CELL COUNT IN PATIENTS WITH CIRRHOSIS AND ASCITES AND ITS PROGNOSTIC VALUE}

Parimita Barua, Krishnasamy Narayanasamy*. Madras Medical College, India

\subsection{6/gutjnl-2021-IDDF.75}

Background Spontaneous bacterial peritonitis (SBP) is defined as ascitic fluid (AF) infection without a surgically treatable intra-abdominal source of infection with or without a positive $\mathrm{AF}$ culture and $\mathrm{AF}$ polymorphonuclear(PMN)cell count of $>250$. In this study, we assessed the prognostic value of $\mathrm{AF}$ total cell count and PMN cell count in patients with cirrhosis and ascites and its outcome.

Methods The prospective study included patients with cirrhosis and ascites. Patients biochemical parameters, manual ascitic fluid total cell and PMN count were studied. Based on AF PMN, patients were categorised into, Reactive effusion (RE)AF $\mathrm{PMN}<150$, Neutrophillic effusion(NE)AF PMN 150-250 and SBP-AF PMN $>250$. Patients were followed up at the end of 1 and 6 months to study the outcome (survival/expired). 